\title{
Article \\ Seed-Mediated Preparation of Ag@Au Nanoparticles for Highly Sensitive Surface-Enhanced Raman Detection of Fentanyl
}

\author{
Yazhou Qin * ${ }^{(1)}$, Binjie Wang, Yuanzhao Wu, Jiye Wang, Xingsen Zong and Weixuan Yao * \\ Key Laboratory of Drug Prevention and Control Technology of Zhejiang Province, Zhejiang Police College, \\ 555 Binwen Road, Hangzhou 310053, China; wangbinjie@zjjcxy.cn (B.W.); wuyuanzhao@zjjcxy.cn (Y.W.); \\ wangjiye@zjjcxy.cn (J.W.); zongxingsen@zjjcxy.cn (X.Z.) \\ * Correspondence: yazhouqin@zju.edu.cn (Y.Q.); yaoweixuan@zjjcxy.cn (W.Y.)
}

\section{check for} updates

Citation: Qin, Y.; Wang, B.; Wu, Y.; Wang, J.; Zong, X.; Yao, W. Seed-Mediated Preparation of Ag@Au Nanoparticles for Highly Sensitive Surface-Enhanced Raman Detection of Fentanyl. Crystals 2021 11,769. https://doi.org/10.3390/ cryst11070769

Academic Editors: Raghvendra Singh Yadav, Anju and Kottakkaran Sooppy Nisar

Received: 8 June 2021

Accepted: 26 June 2021

Published: 1 July 2021

Publisher's Note: MDPI stays neutra with regard to jurisdictional claims in published maps and institutional affiliations.

Copyright: (c) 2021 by the authors. Licensee MDPI, Basel, Switzerland. This article is an open access article distributed under the terms and conditions of the Creative Commons Attribution (CC BY) license (https:// creativecommons.org/licenses/by/ $4.0 /)$.
Abstract: Bimetallic nanoparticles have received extensive attention due to their unique physical and chemical properties, including enhanced optical properties, chemical stability, and better catalytic activity. In this article, we have successfully achieved the controllable preparation of Ag@Au nanoparticles via a seed-mediated growth method. By regulating the amount of seeds-silver nanospheres-we realized that Ag@Au nanoparticles gradually changed from spherical to a seaurchin-like structure. The structure and composition of the prepared nanoparticles were characterized via scanning electron microscopy (SEM), transmission electron microscopy (TEM), energy-dispersive X-ray spectroscopy (EDS), and high-angle circular dark field scanning transmission electron microscopy (HAADF-STEM). In addition, we use the prepared Ag@Au nanoparticles as a substrate material for highly sensitive surface-enhanced Raman spectroscopy (SERS). Using 4-aminothiophenol (4-ATP) as the test molecule, we explored the SERS enhancement effects of Ag@Au nanoparticles with different structures. Furthermore, we used Ag@Au nanoparticles for SERS to detect the drug fentanyl, and realized the label-free detection of fentanyl, with the lowest detection concentration reaching $10^{-7} \mathrm{M}$. This research not only provides a method for preparing bimetallic Ag@Au nanoparticles with different structures, but also provides a reference for the application of Ag@Au nanoparticles in the field of detection technology.

Keywords: Ag@Au nanoparticle; core-shell structure; sea-urchin-like structure; SERS; fentanyl

\section{Introduction}

Noble metal nanocrystals have received extensive attention due to their unique localized surface plasmon resonance characteristics. A large number of studies have reported the application of noble metal nanocrystals in the fields of sensing, catalysis, and detection [1-5]. The characteristics of metal nanomaterials depend largely on their size, structure, and composition. In the past few decades, much research has been devoted to the controllable preparation of noble metal nanocrystals with uniform morphology and size. Various preparation methods have been developed one after another, including the solvothermal method [6-8], electrochemical method [9,10], and photochemical method [11]. Many metal nanocrystals with various regular morphologies have also been prepared. For example, Mirkin et al. realized the transformation of silver nanocrystals from spherical to icosahedral [12]. Yan et al. successfully prepared branched gold nanocrystals with tunable local surface plasmon resonance (LSPR) characteristics, and used the prepared gold nanocrystals as a substrate to achieve quantitative detection of the heme concentration in the cytosol of human red blood cells [13]. By controlling the kinetics involved in the growth of preformed Rh cube seeds, Xia et al. achieved the synthesis of Rh nanocrystals, including cubes, cubic octahedrons, and octahedrons [14]. In addition to polyhedral nanocrystals with regular morphologies, researchers have found that nanomaterials with sharp protrusions and pores often exhibit specific properties. For example, core-satellite structures and sea-urchin-like 
structures with sharp protrusions promote electromagnetic field enhancement by generating multiple "hot spots". This phenomenon has also aroused the interest of a large number of researchers.

In addition, researchers have found that alloy nanomaterials with different elemental compositions have the advantages of various nanomaterials, and show better performance than single-element nanomaterials in various fields. The preparation and performance of bimetallic nanoparticles is now an exciting research area, because they provide a new way to change the properties of the particles by mixing two metals in one particle. Especially for two kinds of metal nanoparticles with different advantages, we can realize the combination of their advantages through the controllable preparation of bimetallic nanoparticles, and avoid their respective disadvantages. For example, for two metal nanomaterials-gold and silver-studies have shown that silver nanomaterials have a larger excitation windowfrom blue to near-infrared-than gold nanoparticles (from red to near-infrared) [15]. In addition, it can be said that silver is a more effective optical material than gold. Due to its greater scattering contribution, which is related to the real part of its dielectric constant, the surface-enhanced Raman scattering (SERS) signal generated by silver is more than 100 times higher than that of similar gold nanostructures [16]. However, the size and shape of gold nanoparticles are easier to control, and have good chemical resistance-that is, higher biocompatibility [17]. Therefore, silver nanomaterials are used more in in vitro research, while gold nanoparticles are used more in in vivo research [18,19].

In order to make full use of the advantages of gold and silver nanoparticles, and avoid their disadvantages, we urgently need to develop an Ag@Au nanomaterial that can coat the surface of silver nanoparticles with a gold layer, or form an alloy of gold and silver on the surface of silver nanoparticles. In general, alloy nanoparticles are obtained via the simultaneous reduction of two metal precursors $\left(\mathrm{Ag}^{+}\right.$and $\left.\mathrm{Au}^{3+}\right)$. Conversely, the controlled growth of the metal on the preformed colloidal surface produces good results if the inner metal (the metal forming the colloid) has a greater surface free energy than the metal on its surface. However, because the surface free energy of gold is greater than that of silver (1.128 and $0.923 \mathrm{Jm}^{-2}$, respectively), it is easier to grow silver on the surface of gold nanoparticles. In fact, a number of studies have also reported that Au@Ag nanoparticles were obtained by growing silver layers on gold seeds [20,21]. For example, Xia et al. recently achieved regulation of the plasma performance of Au@Ag nanoparticles by adjusting the thickness of the silver layer grown on the surface of gold nanospheres and gold nanorods [22]. Cheng et al. adjusted the concentration of the surfactant cetyltrimethylammonium chloride (CTAC) to precisely control the position of the Ag coating on the convex gold nanoarrows. Three different nanostructures were obtained at low, medium, and high CTAC concentrations-namely, anisotropic coating, intermediate coating, and conformal coating, respectively [23]. Conversely, depositing gold onto silver nanoparticles by reducing gold precursors yields a solid solution of the two metals [24]. In general, the gold precursor will undergo a galvanic replacement reaction with the silver nanoparticles, so that the internal silver atoms are first replaced by silver ions, and then the silver ions are reduced to silver atoms by the reducing agent in the solution and deposited on the surface, so that the hollow structure is finally obtained [25-28]. As far as we know, only a few studies have reported the method of growing a gold layer on the surface of silver nanoparticles to prepare Ag@Au core-shell-structured nanoparticles. For example, Xia et al. successfully prepared Ag@Au nuclear sheath nanowires by depositing $\mathrm{Au}$ atoms on the surface of pre-synthesized Ag nanowires, which greatly improved their stability under different corrosive environmental conditions [29]. Kim et al. used sodium sulfite to selectively bind $\mathrm{Au}$ cations, and thereby reduce the reduction potential of $\mathrm{Au}$ $\left(\mathrm{E}^{\circ}=0.111 \mathrm{~V}\right)$. The gold(I) sulfite complex is relatively benign to the Ag nanowire surface, so no oxidative etching will occur; on the contrary, the Au coating is promoted, and finally Ag@Au nanowires are formed [30]. Kim et al. achieved gold-spiked coating of silver particles through controlled Ostwald ripening of small gold nanoparticles on the surface of larger silver particles [31]. 
In this article, in order to fully combine the advantages of gold and silver nanoparticles and avoid their disadvantages, we have developed a method for preparing Ag@Au nanoparticles. Ag@Au nanoparticles have both the excellent surface plasmon resonance characteristics of silver nanoparticles and the stability of gold. First, $\mathrm{Au}^{3+}$ is combined with $\mathrm{Br}^{-}$in CTAB to form $\left[\mathrm{AuBr}_{4}\right]^{-}$, and then the addition of ascorbic acid reduces $\mathrm{Au}^{3+}$ to $\mathrm{Au}^{+}$. Finally, an $\mathrm{Au}(\mathrm{I})-\mathrm{GSH}$ complex is formed by combining $\mathrm{Au}^{+}$with glutathione modified on the surface of silver nanoparticles, and then $\mathrm{Au}^{+}$is reduced by glutathione in situ to grow gold on the surface of silver nanoparticles. By simply adjusting the amount of silver seeds added, we realized the transformation of the $\mathrm{Ag} @$ Au nanoparticle structure from spherical to a sea-urchin-like structure.

\section{Materials and Methods}

\subsection{Materials}

The hydrochloroauric acid trihydrate $\left(\mathrm{HAuCl}_{4} \cdot 3 \mathrm{H}_{2} \mathrm{O}, 99.9 \%\right.$ trace metals basis), hexadecyltrimethylammonium bromide (CTAB, AR, 99\%), glutathione ( $\mathrm{Mw}=307.32, \geq 98 \%)$, 4-aminothiophenol (4-ATP, GC, $\geq 98 \%$ ), nitric acid $\left(\mathrm{HNO}_{3}, \mathrm{AR}\right)$, and hydrochloric acid $(\mathrm{HCl}, \mathrm{AR})$ were purchased from Aladdin. Silver nanospheres (diameter: $80 \mathrm{~nm}$; purity: $>99 \%$ concentration: $0.1 \mathrm{mg} / \mathrm{mL}$, XFJ63) were purchased from Nanjing/Jiangsu XFNANO Materials Tec Co., Let. Fentanyl hydrochloride standard product was purchased from the Shanghai Institute of Criminal Science and Technology (Shanghai, China). All reagents were used directly, without further processing. All of the water used in the experiment was ultrapure water $(18.2 \mathrm{M} \cdot \mathrm{cm}$ ) purified using a Milli-Q Lab System (Nihon Millipore Ltd., Hangzhou, China). The flasks and glass slides used in the experiment were first washed with aqua regia $\left(\mathrm{HCl}: \mathrm{HNO}_{3}=3: 1\right)$ for $30 \mathrm{~min}$, then washed with water $(18.2 \mathrm{M} \Omega \cdot \mathrm{cm})$ and absolute ethanol twice, and finally dried for use.

\subsection{Synthesis of Ag@Au Nanoparticles}

First, we mixed the silver nanoball seeds with $100 \mu \mathrm{L}$ of $1 \mathrm{mM}$ glutathione solution in a $1.5 \mathrm{~mL}$ centrifuge tube at room temperature; using an IKA shaker, we then vortexed it for $1 \mathrm{~min}$, and incubated it for $1 \mathrm{~h}$ to make glutathione modified into silver nanospheres by the Ag-S bond. At the same time, we added $4.0 \mathrm{~mL}$ ultrapure water to a $20 \mathrm{~mL}$ glass flask, followed by $200 \mu \mathrm{L}$ of $10 \mathrm{mM} \mathrm{HAuCl}{ }_{4} \cdot 3 \mathrm{H}_{2} \mathrm{O}$ solution and $0.8 \mathrm{~mL}$ of $0.1 \mathrm{M} \mathrm{CTAB}$ solution. The mixed solution was stirred at $500 \mathrm{rpm}$ for $30 \mathrm{~min}$ at room temperature using an IKA magnetic stirring device, mixed well, and then $475 \mu \mathrm{L}$ of $0.1 \mathrm{M}$ ascorbic acid solution was added, stirring was continued at room temperature for $5 \mathrm{~min}$, and the mixed solution was used as a growth solution. Finally, the silver nanospheres modified with glutathione were added to the growth solution, and the reaction was carried out at room temperature under magnetic stirring for $2 \mathrm{~h}$. The resulting product was centrifuged at $5000 \mathrm{rpm}$ for $10 \mathrm{~min}$; the supernatant was removed, and washed twice with water and ethanol, respectively. In order to explore the effect of the amount of silver nanoball seeds added on the produced Ag@Au NPs, we added $40 \mu \mathrm{L}, 80 \mu \mathrm{L}, 160 \mu \mathrm{L}$, and $500 \mu \mathrm{L}$ of silver nanosphere seeds to the reaction, without changing any of the other reaction conditions.

\subsection{Characterization and SERS Test}

The morphology and structure of the prepared nanoparticles were passed through a field emission scanning electron microscope (SEM, $3.0 \mathrm{kV}$, JEOL, JSM-6700F, Japan), transmission electron microscope (TEM, $100.0 \mathrm{kV}$, JEOL-2100f, Japan), high-resolution TEM (HRTEM, 200 kV, JEOL-2100f, Japan), and selected-area electron diffraction (SAED, JEOL-2100f, Japan) for characterization. The SERS test was conducted using a Raman spectrometer (Thermo Fisher DXR2xi, America) under $633 \mathrm{~nm}$ laser excitation; the laser power was $6 \mathrm{~mW}$, and the acquisition time was $10 \mathrm{~s}$. For surface-enhanced Raman spectroscopy testing, we first added $10 \mu \mathrm{L}$ of Ag@Au nanoparticles to the centrifuge tube, and then added $50 \mu \mathrm{L}$ of 4-ATP solutions of different concentrations, mixing well. The mixed solution was stored at room temperature for $30 \mathrm{~min}$, and then $5 \mu \mathrm{L}$ was dropped on a 
glass slide, dried at room temperature, and used for surface-enhanced Raman spectroscopy (SERS) testing. The SERS test used a laser Raman spectrometer (Thermo Fisher DXR2xi, America) equipped with a microscope $(50 \times$ objective lens) and a CCD detector; the laser source was $633 \mathrm{~nm}$, the acquisition time was $10 \mathrm{~s}$, and the laser power was $6 \mathrm{~mW}$. We used the supporting software to analyze the Raman peak spectrum. The SERS test for the drug fentanyl followed the same process.

\section{Results and Discussion \\ Characterization of Ag@Au NPs}

We studied the morphology and size of Ag@Au nanoparticles via SEM and TEM. Figure $1 \mathrm{a}$ is the SEM image of Ag@Au nanoparticles prepared when the amount of silver seed added is $40 \mu \mathrm{L}$, and Figure $1 \mathrm{~b}$ is the corresponding TEM image. As we can see, the surface of the prepared Ag@Au nanoparticles is distributed with dense, thorn-like structures. From the HRTEM image (Figure 1c) of the gold thorns grown on the surface, it can be seen that the lattice spacing is $0.24 \mathrm{~nm}$, indicating that the exposed crystal faces of the gold thorns are mainly composed of $\{111\}$ planes [4]. Figure $1 \mathrm{~d}$ shows the corresponding HAADF map. We performed EDS characterization of the prepared Ag@Au nanoparticles, as shown in Figure 1e-g. Figure $1 \mathrm{e}, \mathrm{f}$ are the distribution diagrams of silver and gold, respectively. Figure $1 \mathrm{~g}$ shows the overlapping distribution of the two elements. From the EDS results, it can be seen that the atomic ratio of gold to silver is 68:32.
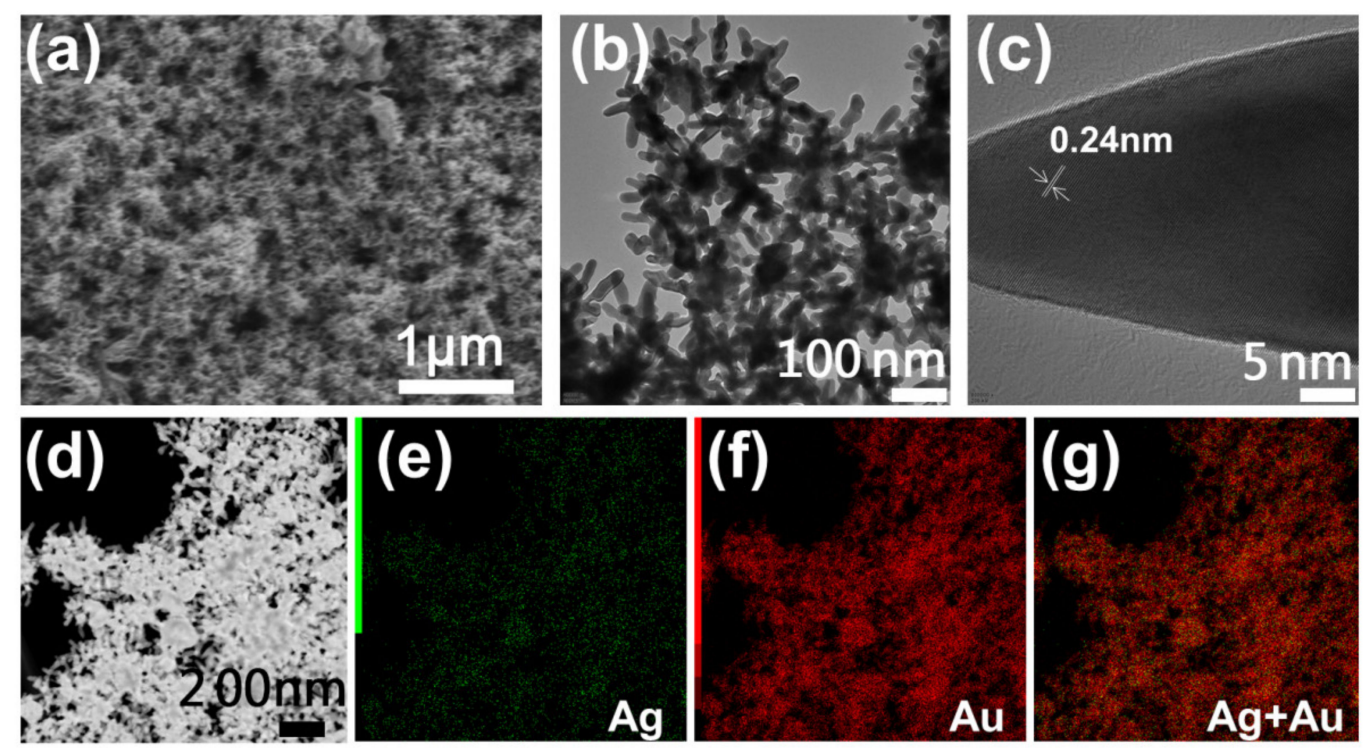

Figure 1. (a) SEM, (b) TEM, (c) HRTEM, and (d) HAADF images of as-prepared Ag@Au NPs, when the amount of Ag seed was $40 \mathrm{uL}$. (e-g) The corresponding EDS images.

Figure 2 shows the characterization results of Ag@Au nanoparticles obtained when the amount of silver seed added is $500 \mu \mathrm{L}$. Figure 2a,b show the SEM and TEM images of Ag@Au nanoparticles, respectively. From the figure, we can clearly see that the Ag@Au nanoparticles obtained still maintain a spherical structure, and have a uniform size distribution of about $95 \mathrm{~nm}$. From its HRTEM image (Figure 2c), we can see that the lattice spacing of gold nanoparticles distributed on the surface is $0.24 \mathrm{~nm}$, indicating that it is mainly composed of $\{111\}$ planes. In addition, from the HAADF map (Figure 2d), it can be seen that only a small part of the Ag@Au nanoparticles are gray in the central area, indicating that the Ag@Au nanoparticles have formed a partially hollow structure, while the main part is still a solid structure. Furthermore, we collected the distribution of Au and Ag elements in Ag@Au nanoparticles via EDX, as shown in Figure 2e-g. From the figure, we can see that gold and silver form an alloy structure distributed over the entire surface. 

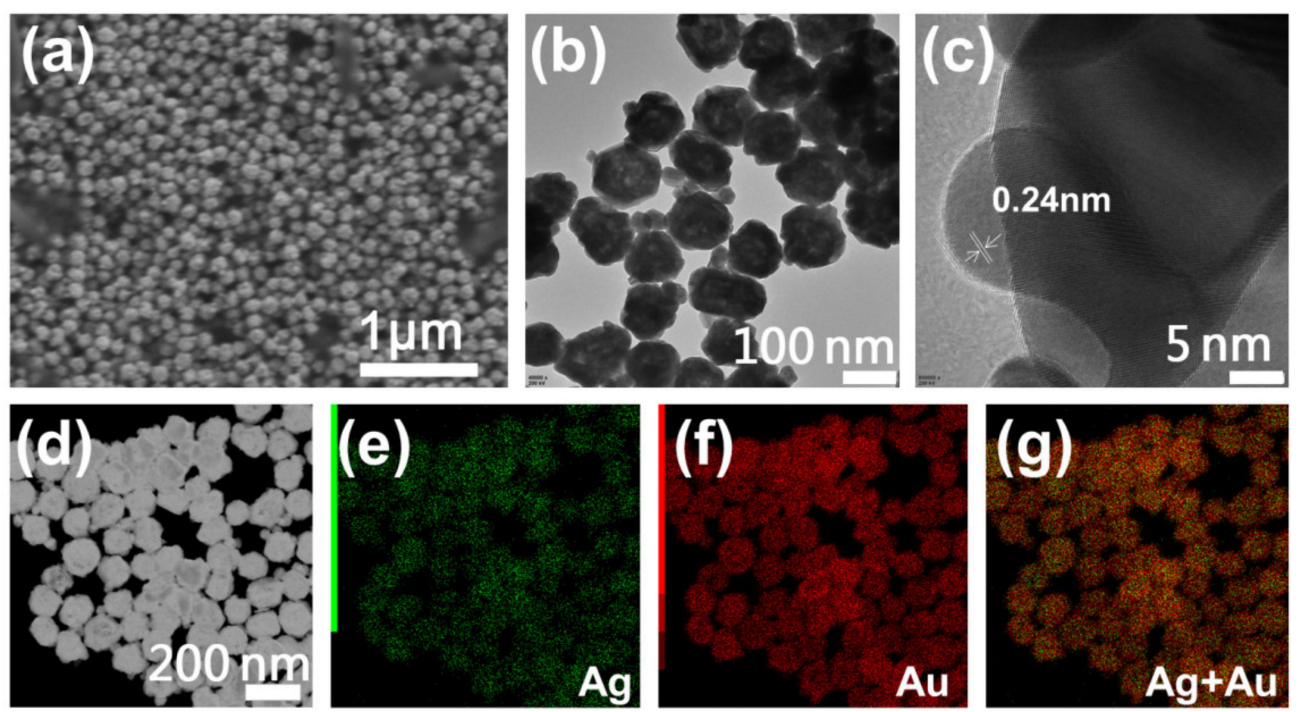

Figure 2. (a) SEM, (b) TEM, (c) HRTEM, and (d) HAADF images of as-prepared Ag@Au nanoparticles, when the amount of Ag seed was $500 \mu \mathrm{L}$. (e-g) The corresponding EDS images.

Furthermore, we explored the effects of the amount of silver seeds on the Ag@Au nanoparticles. As shown in Figure 3, when there was no silver seed, we prepared a mesoporous gold structure (Figure $3 \mathrm{a}$ ). When adding $40 \mu \mathrm{L}$ of silver seeds, we prepared $\mathrm{Ag} @$ Au nanoparticles with dense, thorn-like structures on their surface (Figure $3 b$ ). When the amount of silver seeds was doubled $(80 \mu \mathrm{L})$, the thorn-like structures on the surface of the Ag@Au nanoparticles were reduced (Figure 3c). When the amount of silver seeds was further increased to $160 \mu \mathrm{L}$, the thorn-like structures on the surface were further reduced (Figure 3d). When the amount of silver seed was increased to $500 \mu \mathrm{L}$, we achieved a spherical structure with shape retention (Figure 3e). According to the EDS characterization results of the nanoparticles prepared under different concentrations of silver seeds, we performed a statistical analysis of the proportions of gold and silver atoms, as shown in Figure 3f. The EDS diagrams of Ag@Au nanoparticles prepared under different silver seed conditions are shown in Figure A1. Analyzing the EDS results, when the amount of silver seeds added was 40, 80,160, and $500 \mu \mathrm{L}$, the proportion of tightness in the prepared Ag@Au nanoparticles was $0.68,0.44,0.38$, and 0.20 , respectively. The corresponding proportions of silver were $0.32,0.56,0.62$, and 0.80 , respectively.

Based on the above experimental results, we can make the following inferences on the formation mechanism of $\mathrm{Ag@Au} \mathrm{nanoparticles:} \mathrm{Figure} \mathrm{3g} \mathrm{is} \mathrm{a} \mathrm{schematic} \mathrm{diagram} \mathrm{of}$ the growth of $\mathrm{Ag@Au} \mathrm{nanoparticles.} \mathrm{First,} \mathrm{Au}^{3+}$ ions in $\mathrm{HAuCl}_{4} \cdot 3 \mathrm{H}_{2} \mathrm{O}$ solution combine with $\mathrm{Br}^{-}$ions in $\mathrm{CTAB}$ to form $\left[\mathrm{AuBr}_{4}\right]^{-}$, and then ascorbic acid reduces $\left[\mathrm{AuBr}_{4}\right]^{-}$to get $\mathrm{Au}^{+}$. This process is consistent with previous reports [32,33]. When silver seeds modified with glutathione were added, $\mathrm{Au}^{+}$reduced glutathione adsorbed on the surface of silver seeds in situ to form $\mathrm{Au}^{0}$. It can be seen from Figure 2D that $\mathrm{Au}^{+}$inevitably had a galvanic replacement reaction with silver seeds (forming few hollow structures). However, the majority of Ag@Au nanoparticles are still solid, which also indicates that the glutathione on the surface of the silver seeds largely inhibits the galvanic replacement reaction. In addition, the reduction of glutathione also causes the gold ions to be reduced to gold atoms and deposited on the surface of the silver seeds, which also prevents the occurrence of the galvanic replacement reaction. Thus, when the amount of silver seeds is low, there are enough gold atoms to grow along the direction of glutathione, and eventually form seaurchin-like Ag@Au nanoparticles. When the amount of silver seeds is greater, the Ag@Au spherical shell structure with the original shape will be formed. The above results show that we have successfully prepared Ag@Au nanoparticles. Compared with the previously reported Au@Ag core-shell structure and the Ag@Au hollow structure prepared by the 
displacement reaction, the Ag@Au nanoparticles we prepared have a solid structure. A gold thorn structure grows on the surface of the seed, which realizes the combination of the advantages of gold and silver nanoparticles.
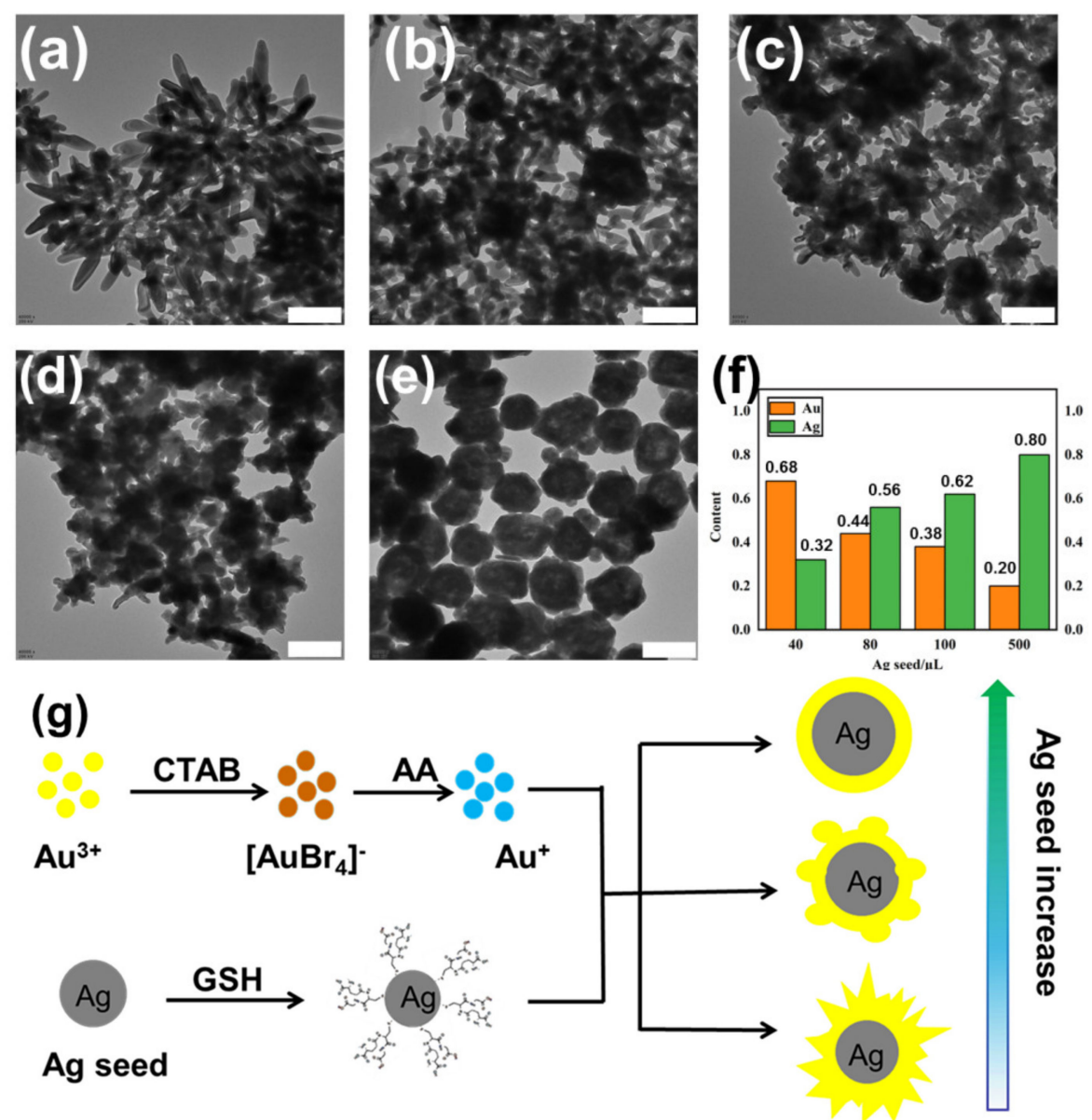

Figure 3. TEM images of as-prepared Ag@Au nanoparticles, when the amount of Ag seed added was (a) $0 \mu \mathrm{L}$, (b) $40 \mu \mathrm{L}$, (c) $80 \mu \mathrm{L}$, (d) $160 \mu \mathrm{L}$, and (e) $500 \mu \mathrm{L}$. (f) A statistical graph of the atomic ratio of $\mathrm{Ag}$ and $\mathrm{Au}$ in $\mathrm{Ag} @ \mathrm{Au}$ nanoparticles prepared with different amounts of silver seeds, according to the EDS results. (g) Schematic diagram of the growth process of Ag@Au nanoparticles. Scale bar: $100 \mathrm{~nm}$.

Furthermore, we used the prepared Ag@Au nanoparticles as the substrate material for surface-enhanced Raman spectroscopy (SERS) for highly sensitive detection of the drug fentanyl. First, we explored the SERS enhancement effect of Ag@Au nanoparticles with different structures. Figure 4 shows the SERS spectra obtained with $10^{-4} \mathrm{M}$ $p$-aminothiophenol (4-ATP) as the test molecule and Ag@Au nanoparticles with different structures as the base material. We can see that the order of SERS enhancement effects, from high to low, is spherical Ag@Au nanoparticles, sea-urchin-shaped Ag@Au nanoparticles, and mesoporous gold nanoparticles. Therefore, in the follow-up test, we use spherical Ag@Au nanoparticles as the enhancement reagent. The Raman characteristic peaks of 4-ATP molecules are located at $1076 \mathrm{~cm}^{-1}$ and $1585 \mathrm{~cm}^{-1}$ [34], and we see obvious SERS peaks at $1140 \mathrm{~cm}^{-1}, 1389 \mathrm{~cm}^{-1}$, and $1432 \mathrm{~cm}^{-1}$, which can be attributed to $\beta \mathrm{CH}+\nu \mathrm{CN}$, $v \mathrm{NN}+\nu \mathrm{CN}$, and $v \mathrm{NN}+\beta \mathrm{CH}$ of $p, p^{\prime}$-dimercaptoazobenzene (DMAB), respectively [4]. This indicates that the prepared nanomaterials have catalytic activity, so that 4-ATP is partially converted to DMAB by catalytic oxidation. 


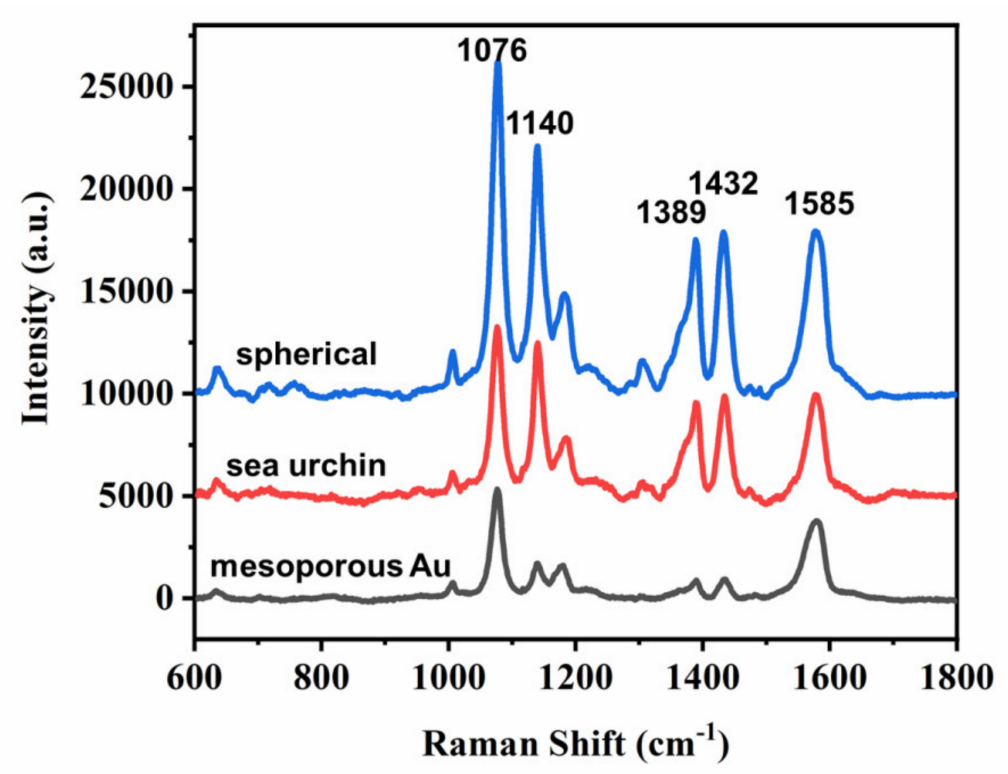

Figure 4. SERS spectra of $10^{-4} \mathrm{M}$ 4-ATP molecules with different substrate materials.

We used spherical Ag@Au nanoparticles to detect fentanyl. Figure 5a shows the Raman spectrum and SERS spectrum of the fentanyl standard substance. As shown in Figure 5a (black line), the Raman peaks of fentanyl solid powder are located at $621 \mathrm{~cm}^{-1}$, $746 \mathrm{~cm}^{-1}, 831 \mathrm{~cm}^{-1}, 1002 \mathrm{~cm}^{-1}, 1030 \mathrm{~cm}^{-1}, 1201 \mathrm{~cm}^{-1}, 1447 \mathrm{~cm}^{-1}$, and $1600 \mathrm{~cm}^{-1}$, which is consistent with previous reports [35]. However, the peak at $621 \mathrm{~cm}^{-1}$ in the SERS spectrum (red line) disappeared. This is because, for a group, the Raman peak of tensile vibration usually appears in a higher frequency range, and is less affected by the external environment. The Raman peak of deformation vibration is usually located in a lower frequency range, and is sensitive to environmental changes [36]. The peak at $621 \mathrm{~cm}^{-1}$ is the bending vibration of C-C-C [35], which is easily affected by the SERS detection environment, and disappears. We assigned the SERS peak of fentanyl, as shown in Table A1. Furthermore we performed SERS tests on different concentrations of fentanyl, and the results are shown in Figure 5b. We can see that the lowest detection concentration is $10^{-7} \mathrm{M}$.

(a)

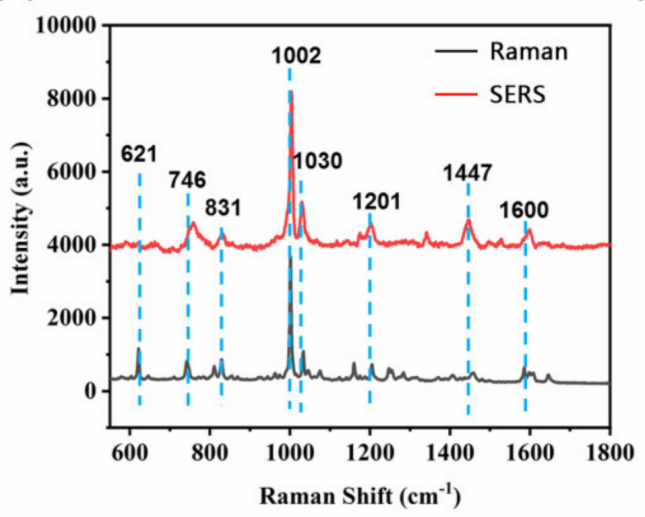

(b)

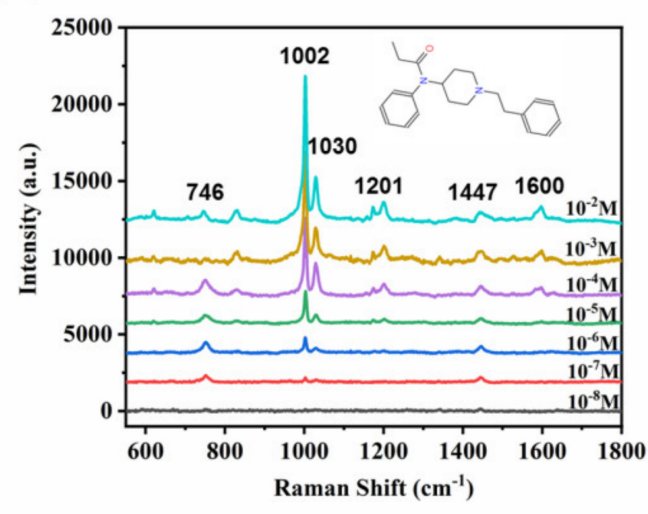

Figure 5. (a) Normal and surface-enhanced Raman spectra for fentanyl $\left(10^{-4} \mathrm{M}\right)$. (b) SERS spectra of fentanyl at different concentrations.

\section{Conclusions}

In this paper, we prepared Ag@Au nanoparticles via a seed-mediated growth method. By adjusting the amount of silver seeds, we realized the transformation of Ag@Au nanopar- 
ticles from a spherical to a sea-urchin-like structure, and proposed the possible growth mechanism. We further explored the SERS enhancement performance of the prepared nanoparticles, using 4-ATP as the test molecule. The results show that the order of SERS enhancement effects, from high to low, is spherical Ag@Au nanoparticles, sea-urchinshaped Ag@Au nanoparticles, and mesoporous gold nanoparticles. Finally, with Ag@Au spherical nanoparticles as the enhancement material, we achieved the highly sensitive SERS detection of fentanyl, with the lowest detection concentration reaching $10^{-7}$.

Author Contributions: Methodology, Y.Q.; validation, W.Y., B.W. and Y.Q.; resources, W.Y., J.W. and X.Z.; data curation, J.W.; writing-original draft preparation, Y.Q.; writing-review and editing, Y.W., B.W. and Y.Q.; supervision, X.Z.; project administration, J.W. and W.Y.; funding acquisition, W.Y. All authors have read and agreed to the published version of the manuscript.

Funding: This research was funded by "the National Key Research and Development Program of Zhejiang Province, grant number 2021C03135", "the National Key Research and Development Program of China Public Safety Risk Prevention and Control, grant number 2018YFC0807201", Zhejiang Police College scientific research project, grant number 2019XJY001 and "Zhejiang Provincial Natural Science Foundation of China under grant number, LGF20C090001" The APC was funded by the National Key Research and Development Program of Zhejiang Province.

Institutional Review Board Statement: The Institutional Review Committee has informed and agreed to the publication of this article.

Informed Consent Statement: All authors know and agree to the publication of this article.

Data Availability Statement: The data presented in this study are available within the article.

Acknowledgments: We would like to express our heartfelt thanks to Mao Zhanpeng for his assistance in the electron microscope test and the assistance provided by XFNANO with the experimental materials.

Conflicts of Interest: The authors declare no conflict of interest.

\section{Appendix A}
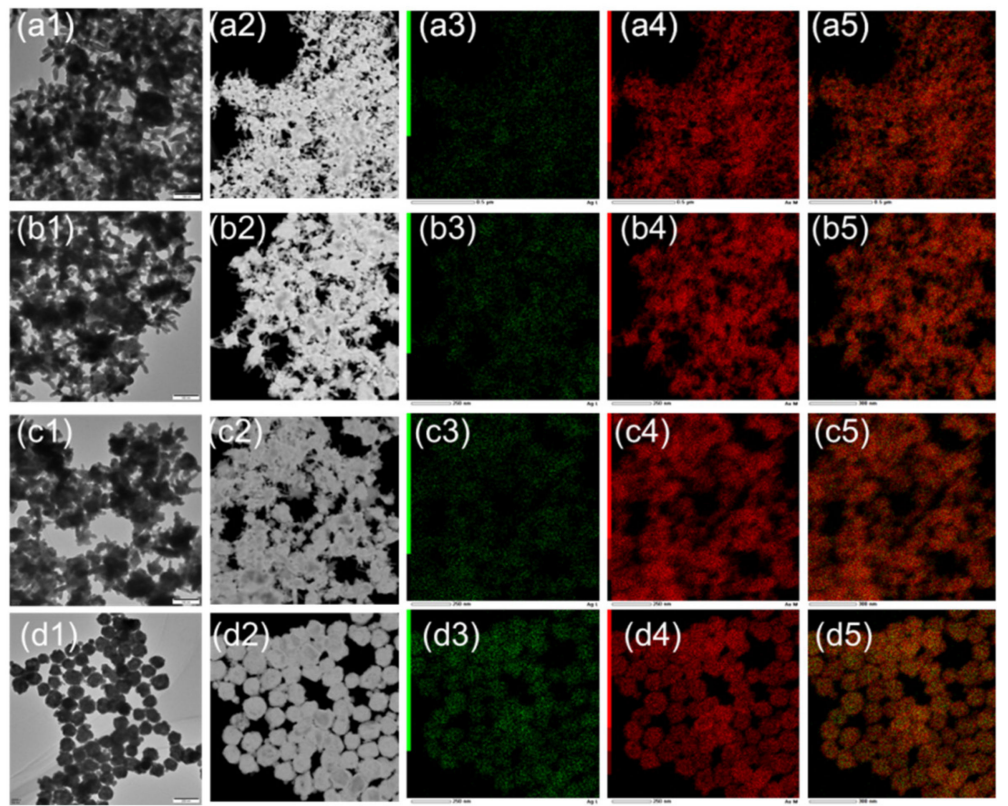

Figure A1. TEM, HAADF, and EDS characterization of the prepared nanoparticles with different amounts of Ag seeds: (a1-a5) $40 \mu \mathrm{L}$, (b1-b5) $80 \mu \mathrm{L},(\mathbf{c 1 - c 5}) 160 \mu \mathrm{L}$, and (d1-d5) $500 \mu \mathrm{L}$. 
Table A1. Characteristic vibrations of fentanyl.

\begin{tabular}{cc}
\hline Fentanyl & \\
\hline 746 & $\delta(\mathrm{C}-\mathrm{C}-\mathrm{C}), \delta\left(\mathrm{N}-\mathrm{CH}_{3}\right)$ \\
\hline 831 & $\gamma(\mathrm{N}-\mathrm{C}-\mathrm{C}-\mathrm{C})$ \\
\hline 1002 & $\delta(\mathrm{CH}) \mathrm{Ar}$ \\
\hline 1030 & $\gamma(\mathrm{C}=\mathrm{C}), \delta\left(\mathrm{CH}_{2}\right)$ twisting in aliphatic ring \\
\hline 1201 & $\delta(\mathrm{C}-\mathrm{C})$ benzyl stretch \\
\hline 1447 & $\delta\left(\mathrm{CH}_{2}\right)$ scissoring \\
\hline 1600 & $v(\mathrm{C}=\mathrm{C})$ Ar \\
\hline
\end{tabular}

\section{References}

1. Ding, S.Y.; Yi, J.; Li, J.F.; Ren, B.; Wu, D.Y.; Panneerselvam, R.; Tian, Z.Q. Nanostructure-based plasmon-enhanced Raman spectroscopy for surface analysis of materials. Nat. Rev. Mater. 2016, 1, 16021-16036. [CrossRef]

2. Li, J.F.; Zhang, Y.J.; Ding, S.Y.; Panneerselvam, R.; Tian, Z.Q. Core-Shell Nanoparticle-Enhanced Raman Spectroscopy. Chem. Rev. 2017, 117, 5002-5069. [CrossRef]

3. Dong, D.; Yap, L.W.; Smilgies, D.M.; Si, K.J.; Shi, Q.; Cheng, W. Two-dimensional gold trisoctahedron nanoparticle superlattice sheets: Self-assembly, characterization and immunosensing applications. Nanoscale 2018, 10, 5065-5071. [CrossRef]

4. Qin, Y.; Lu, Y.; Pan, W.; Yu, D.D.; Zhou, J.G. One-pot synthesis of hollow hydrangea Au nanoparticles as a dual catalyst with SERS activity for in situ monitoring of a reduction reaction. RSC Adv. 2019, 9, 10314-10319. [CrossRef]

5. Ha, M.J.; Kim, J.H.; You, M.; Li, Q.; Fan, C.H.; Nam, J.M. Multicomponent Plasmonic Nanoparticles: From Heterostructured Nanoparticles to Colloidal Composite Nanostructures. Chem. Rev. 2019, 119, 12208-12278. [CrossRef]

6. Qin, Y.; Pan, W.F.; Yu, D.D.; Lu, Y.X.; Wu, W.H.; Zhou, J.G. Stepwise evolution of Au micro/nanocrystals from an octahedron into a truncated ditetragonal prism. Chem. Commun. 2018, 54, 3411-3414. [CrossRef]

7. Qin, Y.Z.; Lu, Y.X.; Yu, D.D.; Zhou, J.G. Controllable synthesis of Au nanocrystals with systematic shape evolution from an octahedron to a truncated ditetragonal prism and rhombic dodecahedron. CrystEngComm 2019, 21, 5602-5609. [CrossRef]

8. Litti, L.; Reguera, J.; Abajo, F. Manipulating chemistry through nanoparticle Morphology. Nanoscale Horiz. 2020, 5, 102-108. [CrossRef]

9. Tian, N.; Zhou, Z.Y.; Yu, N.F.; Wang, L.Y.; Sun, S.G. Direct electrodeposition of tetrahexahedral Pd nanocrystals with high-index facets and high catalytic activity for ethanol electrooxidation. J. Am. Chem. Soc. 2010, 132, 7580-7581. [CrossRef]

10. Du, J.H.; Sheng, T.; Xiao, C.; Tian, N.; Xiao, J.; Xie, A.; Liu, S.; Zhou, Z.; Sun, S.G. Shape transformation of $\{$ hk0\}-faceted Pt nanocrystals from a tetrahexahedron into a truncated ditetragonal prism. Chem. Commun. 2017, 22, 3236-3238. [CrossRef] [PubMed]

11. Guillerm, G.R.; Pablo, D.N.; Antonio, R.; Alejandro, P.; Gloria, T.; Jesús, G.; Luis, B.; Pablo, L.; Luis, G.M.; Mauricio, A.P.; et al. Femtosecond laser reshaping yields gold nanorods with ultranarrow surface plasmon resonances. Science 2017, 358, 640-644.

12. Langille, M.R.; Zhang, J.; Personick, M.L.; Li, S.; Mirkin, C.A. Stepwise evolution of spherical seeds into 20-fold twinned icosahedra. Science 2012, 337, 954-957. [CrossRef] [PubMed]

13. Cai, Z.; Hu, Y.; Sun, Y.; Gu, Q.; Wu, P.; Cai, C.; Yan, Z. Plasmonic SERS Biosensor Based on Multibranched Gold Nanoparticles Embedded in Polydimethylsiloxane for Quantification of Hematin in Human Erythrocytes. Anal. Chem. 2021, 93, 1025-1032. [CrossRef] [PubMed]

14. Zhao, M.; Chen, Z.; Shi, Y.; Hood, Z.D.; Lyu, Z.; Xie, M.; Chi, M.; Xia, Y. Kinetically Controlled Synthesis of Rhodium Nanocrystals with Different Shapes and a Comparison Study of Their Thermal and Catalytic Properties. J. Am. Chem. Soc. 2021, 143, 6293-6302. [CrossRef] [PubMed]

15. Zhao, J.; Pinchuk, A.O.; McMahon, J.M.; Li, S.; Ausman, L.K.; Atkinson, A.L.; Schatz, G.C. Methods for Describing the Electromagnetic Properties of Silver and Gold Nanoparticles. Acc. Chem. Res. 2008, 41, 1710-1720. [CrossRef]

16. Abajo, F.J. Colloquium: Light scattering by particle and hole arrays. Rev. Mod. Phys. 2007, 79, 1267-1290. [CrossRef]

17. Murphy, C.J.; Gole, A.M.; Stone, J.W.; Sisco, P.N.; Alkilany, A.M.; Goldsmith, E.C.; Baxter, S.C. Gold Nanoparticles in Biology: Beyond Toxicity to Cellular Imaging. Acc. Chem. Res. 2008, 41, 1721-1730. [CrossRef] [PubMed]

18. Schlücker, S. Surface-enhanced Raman spectroscopy: Concepts and chemical applications. Angew. Chem. Int. Ed. 2014, 53, 4756-4795. [CrossRef]

19. Sperling, R.A.; Gil, P.R.; Zhang, F.; Zanella, M.; Parak, W.J. Biological applications of gold nanoparticles. Chem. Soc. Rev. 2008, 37, 1896-1908. [CrossRef]

20. Guisbiers, G.; Mendoza-Cruz, R.; Bazán-Díaz, L.; Elázquez-Salazar, J.J.; Mendoza-Perez, V.R.; Torres, J.A.; Lopez, J.L.; Carrizales, J.M.; Whetten, R.L.; Yacamán, M.J. Response to "Comment on 'Electrum, the Gold-Silver Alloy, from the Bulk Scale to the Nanoscale: Synthesis, Properties, and Segregation Rules'”. ACS Nano 2016, 10, 188-198. [CrossRef] [PubMed] 
21. Cardinal, M.F.; González, B.R.; Puebla, R.A.; Juste, J.P.; Marzán, L.M. Modulation of Localized Surface Plasmons and SERS Response in Gold Dumbbells through Silver Coating. J. Phys. Chem. C 2010, 114, 10417-10423. [CrossRef]

22. Gao, Z.; Shao, S.; Gao, W.; Tang, D.; Tang, D.; Zou, S.; Kim, M.J.; Xia, X. Morphology-Invariant Metallic Nanoparticles with Tunable Plasmonic Properties. ACS Nano 2021, 15, 2428-2438. [CrossRef]

23. Dong, D.; Shi, Q.; Sikdar, D.; Zhao, Y.; Liu, Y.; Fu, R.; Premaratne, M.; Cheng, W. Site-specific Ag coating on concave Au nanoarrows by controlling the surfactant concentration. Nanoscale Horiz. 2019, 4, 940-946. [CrossRef]

24. Liao, H.; Fisher, A.; Xu, Z.J. Surface Segregation in Bimetallic Nanoparticles: A Critical Issue in Electrocatalyst Engineering. Small 2015, 11, 3221-3246. [CrossRef]

25. Sun, Y.; Mayers, B.T.; Xia, Y. Template-Engaged Replacement Reaction: A One-Step Approach to the Large-Scale Synthesis of Metal Nanostructures with Hollow Interiors. Nano Lett. 2002, 2, 481-485. [CrossRef]

26. Sun, Y.; Xia, Y. Shape-Controlled Synthesis of Gold and Silver Nanoparticles. Science 2002, 298, 2176-2179. [CrossRef]

27. Xuan, H.; Nguyen, D.D.; Thi, T.H.P.; Nguyen, V.T.; Nguyen, X.C.; Vu, V.T. Tunable LSPR of silver/gold bimetallic nanoframes and their SERS activity for methyl red detection. RSC Adv. 2021, 11, 14596-14606.

28. Ahn, J.; Wang, D.; Ding, Y.; Zhang, J.; Qin, D. Site-Selective Carving and Co-Deposition: Transformation of Ag Nanocubes into Concave Nanocrystals Encased by Au-Ag Alloy Frames. ACS Nano 2017, 12, 298-307. [CrossRef] [PubMed]

29. Yang, M.; Hood, Z.D.; Yang, X.; Chi, M.; Xia, Y. Facile synthesis of Ag@Au core-sheath nanowires with greatly improved stability against oxidation. Chem. Commun. 2017, 53, 1965-1968. [CrossRef]

30. Choi, S.; Han, S.I.; Jung, D.; Hwang, H.J.; Lim, C.; Bae, S.; Park, O.K.; Tschabrunn, C.M.; Lee, M.; Bae, S.Y.; et al. Highly conductive, stretchable and biocompatible $\mathrm{Ag}-\mathrm{Au}$ core-sheath nanowire composite for wearable and implantable bioelectronics. Nat. Nanotech. 2018, 13, 1748-3387. [CrossRef]

31. Irene, C.; Ramon, A.A.; Nicolas, P.P. Gold-spiked coating of silver particles through cold nanowelding. Nanoscale 2021, 13, 4530-4536.

32. Lee, H.; Ahn, H.Y.; Mun, J.; Lee, Y.Y.; Kim, M.; Cho, N.H.; Chang, K.; Kim, W.S.; Rho, J.; Nam, K. Amino-acid-and peptide-directed synthesis of chiral plasmonic gold nanoparticles. Nature 2018, 556, 360-365. [CrossRef] [PubMed]

33. Qin, Y.Z.; Wu, Y.; Wang, B.; Wang, J.; Zong, X.; Yao, W. Controllable preparation of sea urchin-like Au NPs as a SERS substrate for highly sensitive detection of the toxic atropine. RSC Adv. 2021, 11, 19813-19818. [CrossRef]

34. Huang, Y.F.; Zhu, H.P.; Liu, G.K.; Wu, D.Y.; Ren, B.; Tian, Z.Q. When the signal is not from the original molecule to be detected: Chemical transformation of para-aminothiophenol on Ag during the SERS measurement. J. Am. Chem. Soc. 2010, 132, 9244-9246. [CrossRef] [PubMed]

35. Haddad, A.; Greencand, O.; Lombardi, J.R. Detection of fentanyl in binary mixtures with cocaine by use of surface-enhanced Raman spectroscopy. Spectrosc. Lett. 2019, 8, 462-472. [CrossRef]

36. Matas, M.D.; Edwards, H.G.; Lawson, E.E.; Shields, L.; York, P. FT-Raman spectroscopic investigation of a pseudopolymorphic transition in caffeine hydrate. J. Mol. Struct. 1998, 440, 97-104. [CrossRef] 